Document downloaded from:

http://hdl.handle.net/10251/60813

This paper must be cited as:

Medina Sevila, P.; Almenar Terre, V.; Corral, JL. (2015). Combined data detection scheme for zero-padded OFDM signals in MMF links. IEEE Photonics Technology Letters. 27(16):1753-1756. doi:10.1109/LPT.2015.2439158.

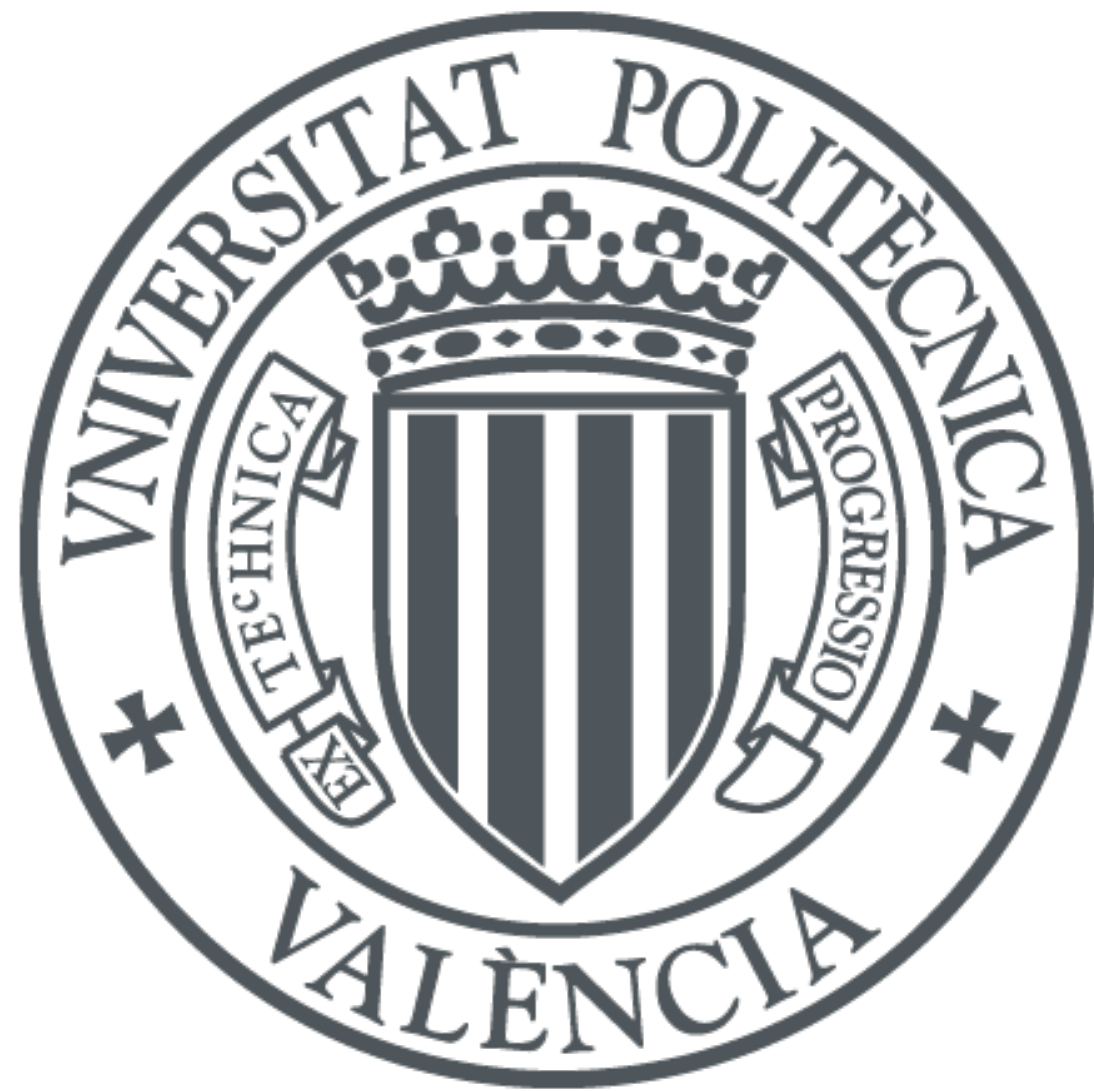

The final publication is available at

http://dx.doi.org/10.1109/LPT.2015.2439158

Copyright Institute of Electrical and Electronics Engineers (IEEE)

Additional Information

(C) (c) 2015 IEEE. Personal use of this material is permitted. Permission from IEEE must be obtained for all other uses, in any current or future media, including reprinting/republishing this material for advertising or promotional purposes, creating new collective works, for resale or redistribution to servers or lists, or reuse of any copyrighted component of this work in other works. 


\title{
Combined Data Detection Scheme for Zero Padded OFDM Signals in MMF Links
}

\author{
Pau Medina, Vicenç Almenar, and Juan Luis Corral
}

\begin{abstract}
In this paper, we propose a receiver scheme for zero padding orthogonal frequency division multiplexing (ZP-OFDM) that combines low complexity from Overlap-and-Add (OLA) equalizer and low error rate provided by successive interference cancellation (SIC) data detection from optimal ordering Vertical Bell Laboratories Layered Space-Time (V-BLAST) architecture. Results of numerical simulations on multi-mode optical fiber links show that the proposed scheme improves the error rate performance of zero forcing equalization $(\mathrm{ZF})$ receiver, reaching results similar to V-BLAST. For example, the proposed scheme can reach $33.9 \mathrm{~Gb} / \mathrm{s}$ in a $600 \mathrm{~m}$ link, whereas the $\mathrm{ZF}$ receiver would reach $29.06 \mathrm{~Gb} / \mathrm{s}$ and cyclic prefix OFDM only $19.37 \mathrm{~Gb} / \mathrm{s}$. These results are obtained with a reduction in computational complexity (measured in number of real products) of $86 \%$ in detection and $66 \%$ in preprocessing with respect to the $\mathrm{ZF}$ receiver, and $44 \%$ and $86 \%$ with respect to the V-BLAST receiver.
\end{abstract}

Index Terms - Digital signal processing, equalizers, OFDM modulation, optical fiber communication, multimode fiber.

\section{INTRODUCTION}

$\mathrm{O}$ RTHOGONAL frequency division multiplexing (OFDM) uses the spectrum efficiently and is able to compensate dispersion impairments adding a guard interval to each transmitted block. These advantages have been exploited to implement OFDM over fiber optics systems.

The most commonly used guard interval is the cyclic prefix (CP-OFDM), where the last samples of each OFDM symbol are copied and added in front of the OFDM symbol. At the receiver, $\mathrm{CP}$ samples are discarded as they can be interfered by last samples from the previous OFDM symbol, then each block of samples is processed by a Discrete Fourier Transform (DFT) and, finally, equalization is performed by a single-tap on each carrier. Low complexity reception is attained at the expense of the redundancy introduced by $\mathrm{CP}$.

Optical CP-OFDM can successfully compensate chromatic dispersion induced by single-mode optical fiber (SMF) links [1], or modal dispersion associated to multi-mode optical fiber (MMF) links [2]. However, there is some optical scenarios characterized by strong frequency selectivity where performance of CP-OFDM is reduced. Zero padded OFDM (ZP-OFDM), where the guard interval is composed of zero

This work was supported by the Spanish Ministerio de Economia y Competitividad under projects TEC2012-38558-C02-02 and TEC2012-38558C02-01. The work of P. Medina was supported by the Formación de Personal Investigador grant program of the Universitat Politècnica de València. samples, has been proposed to overcome this limitation: in single mode fiber (SMF) links it provides higher tolerance to polarization-mode dispersion (PMD) [3] and in MMF links it increases the link capacity or coverage [4].

Whereas channel equalization in CP-OFDM has low complexity, ZP-OFDM makes use of a zero forcing (ZF) equalizer which increases the receiver complexity due to the matrix inversion required [6]. There also exists a low-complexity equalization implementation for ZP-OFDM called Overlap-and-Add (OLA) [6], which is equivalent to $\mathrm{CP}-\mathrm{OFDM}$ in performance and complexity.

In addition, as the received ZP-OFDM signal is affected by inter-carrier interference (ICI), it is possible to use successive interference cancellation (SIC) schemes to equalize the received signal in a similar way as it is done to cancel interference between different transmitters in multiple-input multiple-output (MIMO) systems. For instance, a suboptimal SIC scheme has presented better error rate performance than ZF for ZP-OFDM in SMF links with PMD [3].

In this paper we investigate the use of the optimum SIC scheme, known as Vertical Bell Laboratories Layered Space-Time architecture (V-BLAST) that was originally developed for MIMO systems [7], to equalize the ZP-OFDM signal for optimizing error rate performance. However, as the complexity associated to V-BLAST makes implementation difficult in practice, we propose a combined reception scheme: OLA detection will be used to detect data associated to less attenuated carriers and, after cancelling the signal contribution due to OLA detected data, V-BLAST architecture will be applied to the most unreliable subcarriers in order to deal with a reduced order system. The main aim of this proposal is to achieve the better error rate performance given by V-BLAST, but with the minimum possible complexity. Performance of OLA+V-BLAST will be evaluated over MMF links by means of numerical simulations showing results of error probability and receiver complexity associated to the combined scheme compared with OLA, ZF and V-BLAST.

The organization of this paper is as follows. In Section II we describe the signal model of different ZP-OFDM receiver schemes and their estimated complexity. Section III defines the simulation model for the MMF and shows performance results

V. Almenar and P. Medina are with Instituto de Telecomunicaciones y Aplicaciones Multimedia, Universitat Politècnica de València, Valencia 46022, Spain (e-mail: valmenar@dcom.upv.es; pamese@teleco.upv.es).

J. L. Corral is with Nanophotonics Technology Center, Universitat Politècnica de València, Valencia 46022, Spain (e-mail: jlcorral@dcom.upv.es). 
and computation complexity. Finally, some remarks and conclusions are stated in Section IV. In the following sections ()$^{*},()^{T},()^{H}$ and ()$^{\dagger}$, denote conjugate, matrix transposition, matrix conjugate transposition and Moore pseudo-inverse respectively.

\section{ZP-OFDM SIGNAL RECEIVERS}

This section defines the ZP-OFDM signal model and describes the receiver schemes evaluated in this paper, including an estimate of their computational complexity. This complexity has been divided into two parts: preprocessing and detection. The first one corresponds to the operations done before data detection and performed every time the channel changes, while the second one corresponds to the operations for data estimation done every time a new OFDM symbol arrives. We have expressed computational complexities using the number of real multiplications, as this one is the operation that consumes more area and power in hardware implementations. We have assumed that a complex product is equivalent to four real products.

To generate a real valued OFDM signal, also called discrete multi-tone modulation (DMT) signal, the sequence of $N_{\text {DATA }}$ information symbols carried in each block must keep Hermitian symmetry with no data transported over DC and high frequency carriers:

$\mathbf{s}_{N}=\left[0, s(1), \ldots, s\left(N_{D A T A}\right), 0, \ldots, 0, s^{*}\left(N_{D A T A}\right), \ldots, s^{*}(1)\right]$

The received samples consist of a $P=N+D$ sized column vector (being $N$ the block length and $D$ the size of the guard interval), and can be represented as:

$\mathbf{r}_{Z \mathbf{P}}=\mathbf{H} \cdot \mathbf{F}_{N}^{H} \cdot \mathbf{s}_{N}+\mathbf{w}=\mathbf{G} \cdot \mathbf{s}_{N}+\mathbf{w}$.

Where matrix $\mathbf{F}_{N}$ corresponds to a $N$ points DFT matrix and its transposed conjugation gives the inverse DFT; $\mathbf{H}$ is the convolution matrix which is real valued lower Toeplitz of size $P \times N$, with first column defined by the discrete channel impulse response of order $L:\left[h_{0}, h_{1}, \ldots, h_{L}, 0, \ldots 0\right]^{T} ; \mathbf{w}$ corresponds to $P$ samples of real Gaussian noise; and $\mathbf{G}$ is called the transmission matrix [6].

\section{A. Zero Forcing Receiver}

A ZF receiver can obtain better performance than an OLA receiver because it can exploit the diversity induced by multipath channels [5]. The equalizing matrix is defined as the pseudo-inverse of the transmission channel matrix: $\mathbf{W}=\mathbf{G}^{\dagger}=\mathbf{F}_{\mathrm{N}} \cdot \mathbf{H}^{\dagger}$. The rows of the equalizing matrix $\left(\mathbf{W}_{i}\right)$ form nulling vectors, each row corresponding to a data subcarrier; symbol recovery is always assured as convolution matrix $\mathbf{H}$ is always invertible. Each subcarrier is obtained by $\hat{s}_{N}^{Z F}(i)=\mathbf{W}_{i} \cdot \mathbf{r}_{Z P}$, as described in [6].

Computational complexity associated to preprocessing originates from inverting the transmission matrix, which has a cost of: $(P+1) N^{2}+N^{3} / 3+\left(\log _{2}(N / 2)-3\right) P N-61 / 6 N$ real multiplications. The detection stage is based on vector multiplication and it costs $4 P N_{D A T A}$ real multiplications.

\section{B. V-BLAST Receiver}

V-BLAST reception algorithm consists in detecting the most favorable carrier in each iteration, in terms of carrier interference ratio, and cancelling the contribution of this detected symbol from the received signal to obtain a system with a reduced order. Thereby, the diversity exploited in each iteration is progressively increased and the overall system performance measured in error probability overcomes ZF and OLA receivers, especially for last detected symbols as they correspond to the most attenuated subcarriers.

The V-BLAST scheme described in this work follows the one proposed in [8] for MIMO systems and has been adapted to ZP-OFDM systems. This algorithm benefits from efficient inverse Cholesky factorization and has been proved to be the most efficient implementation in terms of computational complexity.

As commented above, before using this algorithm in ZP-OFDM receivers, it is necessary to change the initialization stage as follows: this algorithm solves normal equations and is initialized in three steps: firstly, the normal matrix $\mathbf{R}_{N}$ is calculated in terms of the DFT matrix and the autocorrelation matrix of channel impulse response $(\Psi)$ as:

$$
\mathbf{R}_{N}=\mathbf{G} \cdot \mathbf{G}^{H}=\mathbf{F}_{N} \cdot\left(\mathbf{H}^{T} \cdot \mathbf{H}\right) \cdot \mathbf{F}_{N}^{H}=\mathbf{F}_{N} \cdot \boldsymbol{\Psi} \cdot \mathbf{F}_{N}^{H} .
$$

Secondly, the signal vector of $N$ samples is calculated as:

$$
\mathbf{z}_{N}=\mathbf{G}^{H} \cdot \mathbf{r}_{Z P}=\mathbf{F}_{N} \cdot \mathbf{H}^{T} \cdot \mathbf{r}_{Z P} .
$$

Finally, this algorithm is applied to a system with order $M=2 N_{D A T A}$ that corresponds only to carriers transporting data, that is to say, it operates with a partition of $M$ rows and columns from $\mathbf{R}_{N}$, and $M$ rows from $\mathbf{s}_{N}$ and $\mathbf{z}_{N}$ to construct a reduced order initial system. For example, $\mathbf{s}_{M}$ is defined as:

$\mathbf{s}_{M}=\left[s(1), s(2), \ldots, s\left(N_{D A T A}\right), s^{*}\left(N_{D A T A}\right), \ldots, s^{*}(1)\right]$.

After this initialization, the rest of the algorithm described in [8] can be employed. The computation complexity of the preprocessing stage has a cost of $P^{2}-2 P N+(1+4 \log 2(N)) N^{2}$

$+P / 2-N / 2+17 / 3 N^{3}{ }_{D A T A}+9 N^{2}$ DATA $+10 / 3 N_{D A T A}-4$ real multiplications, meanwhile, the detection stage costs $P N-N^{2}+N \log _{2}(N / 2)+N^{2}{ }_{D A T A}+4 N_{D A T A}$ real multiplications.

\section{Combined OLA+V-BLAST Receiver}

The low complexity proposed receiver is a combination of two detection schemes: OLA and V-BLAST. In a first stage, the OLA receiver detects those carriers $\left(N_{O L A}\right)$ that have better signal to noise ratio, which correspond to those with higher values in the channel frequency response. After that, OLA detected symbols are used to generate their signal contribution to $\mathbf{r}_{Z P}$ by means of the transmission matrix $\mathbf{G}$ and, then, a 
parallel cancelation is carried out. In a second stage an ICI receiver using the V-BLAST approach commented above is employed over the reduced order problem to detect the remaining subcarriers $\left(N_{V B}\right)$. The number of detected subcarriers fulfills: $N_{O L A}+N_{V B}=N_{D A T A}$.

Thus, taking into account that the V-BLAST problem has been reduced to order $M=2 N_{V B}$, the computation complexity of the preprocessing stage is given by: $(P+1) N \log _{2}(N / 2)+$ $2 N_{D A T A}+14 N_{O L A}+4 P N_{V B}^{2}+4 P N_{V B}+111 / 3 N_{V B}^{3}+36 N_{V B}^{2}$ $+20 / 3 N_{V B}-4$ real multiplications. The detection stage has a cost of $N \log _{2}(N / 2)+4 N_{O L A}+2 P N_{V B}+4 N_{V B}^{2}+8 N_{V B}$ real multiplications.

\section{SimUlation Results}

The model employed in the numerical simulations defines a single $1550 \mathrm{~nm}$ wavelength unamplified intensity modulated and direct detected (IM-DD) optical MMF link and has been previously used to study optical OFDM [4][9]. The transmitter models a directly modulated laser (DML) driven by a DMT signal limited in power to $5 \mathrm{dBm}$ to prevent fiber nonlinearities. The signal is generated by a digital-analog converter (DAC) with 10 bit resolution. The sample rate will be kept at 12.5 Gsamples/s and both the DML and the DAC are assumed to have a $6.25 \mathrm{GHz}$ electrical bandwidth (the same as the OFDM signal) with a flat frequency response. The modulating signal dynamic range is limited by a $13 \mathrm{~dB}$ clipping of the OFDM signal and the DC value is adjusted accordingly. At the receiver, the analog-digital converter (ADC) has 10 bits resolution and $13 \mathrm{~dB}$ clipping, too. The electrical noise is characterized by a 0.8 quantum efficiency and a $20.7 \mathrm{pA} / \mathrm{Hz}^{1 / 2}$ noise power spectral density.

The channel impulse response is modeled as a finite impulse response (FIR) filter where each modal component is associated with a delay and an attenuation value. Equal amplitude 80 modes are simulated, each one contributing to the channel impulse response with a Gaussian-shape pulse of 10 ps width. The delay of each mode is modeled as an independent random variable with uniform distribution around the average delay, with a maximum deviation equal to half the maximum differential mode delay (DMD). This one is given by $\mathrm{DMD}=2 \mathrm{~ns} / \mathrm{km}$ and represents the worst $5 \%$ of installed MMF links operating at $1550 \mathrm{~nm}$ wavelength [4].

The guard interval length is set to $D=N / 4$ of the OFDM symbol length, which corresponds to $1.28 \mathrm{~ns}, 2.56 \mathrm{~ns}$ and 5.12 ns for $N=64,128$ or 256 DFT sizes, respectively. These DFT sizes and their corresponding guard intervals can cope with the maximum DMD expected for fiber lengths of 600 , 1200 and $2400 \mathrm{~m}$, respectively. The real valued DMT signal is obtained modulating DFT positive frequencies subcarriers except DC $\left(N_{D A T A}=31,63\right.$ or 127$)$ and forcing Hermitian symmetry in the remaining subcarriers. Active subcarriers are modulated with the same $L$-QAM modulation level with $L=4$, $8,16,32,64,128$, or 256 , that is, from $B=2$ bits, up to 8 bits per subcarrier. The effective bit rate can be calculated as: $R=B \cdot f_{s} \cdot N_{D A T A} / P$. As the modulation level is increased, the effective bit rate $R$ increases accordingly, $R=9.68,14.53$, $19.37,24.21,29.06,33.90$ and $38.75 \mathrm{~Gb} / \mathrm{s}$ for $N=64$. Finally, note that if the DFT length is changed, the effective bit rate for the same $L$ changes slightly, for example a 64-QAM modulation level gives $29.06,29.53$ or $29.77 \mathrm{~Gb} / \mathrm{s}$ for $N=64$, 128 and 256, respectively.

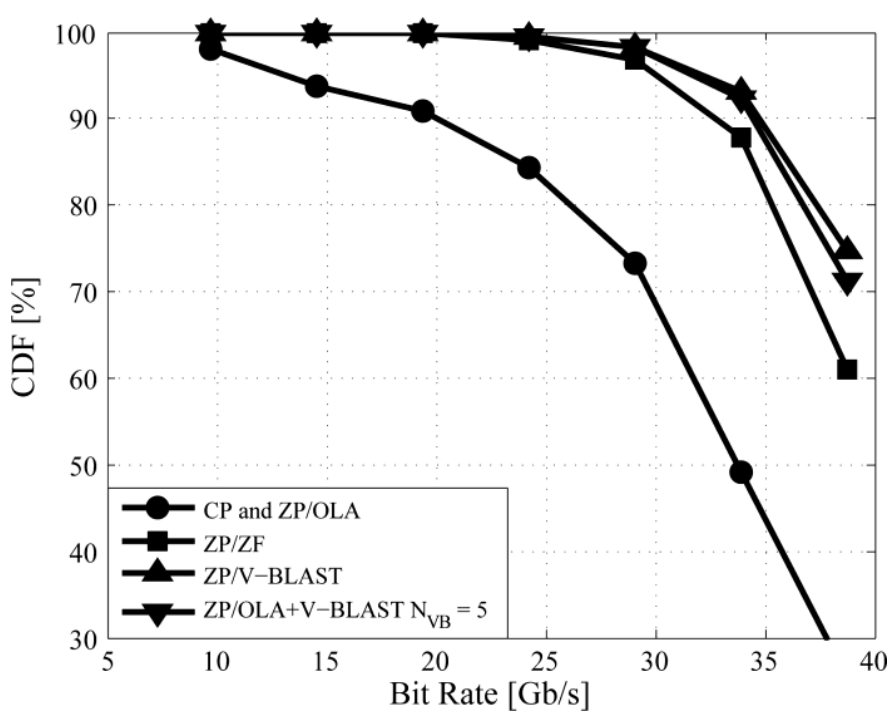

Fig. 1. Cumulative distribution function vs. bit rate curves for $\mathrm{BER}<10^{-3}$, OFDM receivers CP, ZP/OLA, ZP/ZF, ZP/V-BLAST and ZP/OLA+V-BLAST with 64-points FFT, using 1000 realizations of a $600 \mathrm{~m}$ length MMF link.

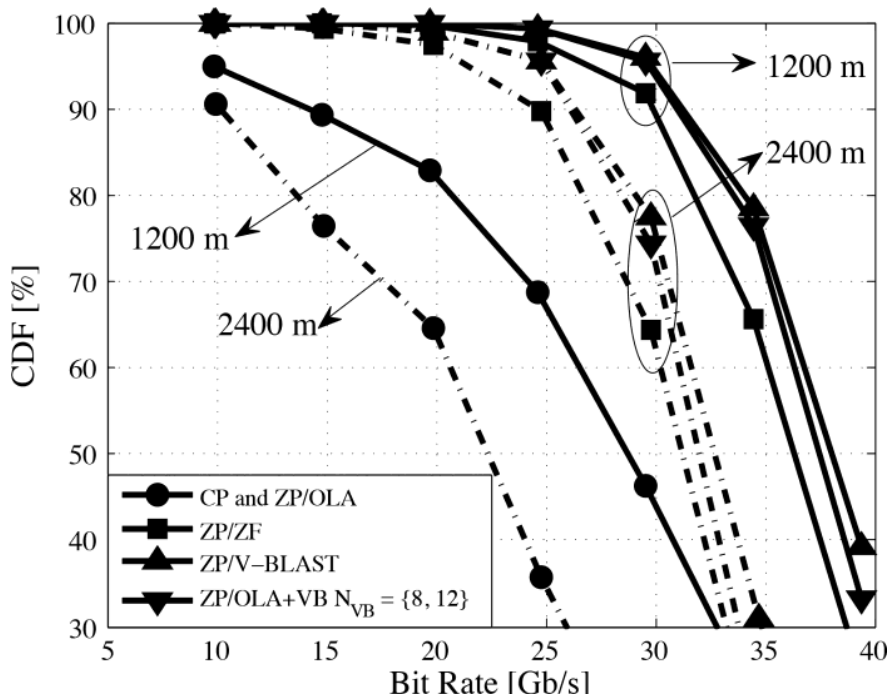

Fig. 2. CDF vs. bit rate curves for $\mathrm{BER}<10^{-3}$ for OFDM receivers $\mathrm{CP}$, ZP/OLA, ZP/ZF, ZP/V-BLAST and ZP/OLA+V-BLAST, with 128 and 256 points DFTs, for 1000 realizations of 1200 and $2400 \mathrm{~m}$ length MMF links.

Fig. 1 shows percentage of cumulative distribution function (CDF) of 1000 different channel realizations that provide a bit error rate (BER) better than $10^{-3}$. Simulations correspond to a link length of $600 \mathrm{~m}$ and an OFDM symbol length of $N=64$. CDF results in Fig. 1 exhibit that the proposed combined receiver detecting only 5 carriers with V-BLAST $\left(N_{V B}=5\right)$ has a performance similar to detecting all carriers with V-BLAST. If we set a CDF threshold of $90 \%$ to choose the modulation order [4][9], the proposed receivers can employ a 128-QAM constellation whereas the ZF receiver should use 64-QAM, this means that it is possible to increment the data rate from $29.06 \mathrm{~Gb} / \mathrm{s}$ up to $33.9 \mathrm{~Gb} / \mathrm{s}$ in more than $99.5 \%$ of all installed MMF links up to $600 \mathrm{~m}$. As expected, conventional CP-OFDM 
offers a quite lower performance, being limited to use 16-QAM $(19.37 \mathrm{~Gb} / \mathrm{s})$ in the same scenario. The performance of the OLA receiver in ZP-OFDM is the same as the one of CP-OFDM. In a similar way, the results for $1200 \mathrm{~m}(N=128)$ and $2400 \mathrm{~m}$ $(N=256)$ are presented in Fig. 2, where $N_{V B}$ is 8 and 12 , respectively. It can be seen in both figures how the performance of the proposed data detection receivers for ZP-OFDM is improved respect to the use of the $\mathrm{ZF}$ receiver proposed in [4]. It is also shown that the combined OLA+V-BLAST receiver can achieve the performance of V-BLAST with low values of $N_{V B}$.

Fig. 3 presents the computational complexity, measured in real products, for preprocessing (a) and detection (b) stages when a DFT size of $N=64$ samples is employed. The computational complexity values have been normalized to ZF receiver complexity. The combined OLA+VBLAST scheme has lower preprocessing complexity than V-BLAST alone if the number of subcarriers detected using V-BLAST is lower than 21 and lower preprocessing complexity than $\mathrm{ZF}\left(10^{\circ}\right.$ reference line in the graph) if $N_{V B} \leq 13$. On the other hand, the threshold level for the detection stage complexity is $N_{V B}=10$ subcarriers and both schemes have always lower complexity than ZF. In both cases, the number of subcarriers $N_{V B}$ used in the simulation shown in Fig. 1 is much lower than these thresholds. Finally, Table I gives a broader comparison of computational complexities. Here, we compare the computational cost of the combined detection scheme, using the $N_{V B}$ values employed in Fig. 1 and 2, with the cost of a ZF receiver and a full V-BLAST receiver. In both cases, detection and preprocessing are less complex if the proposed combined scheme is employed, whereas this difference increases with the size of the DFT. According to these results, when a DFT of 64 samples is used, the combined receiver has a reduction of $86 \%$ in detection and $66 \%$ in preprocessing respect to ZF receiver, reaching savings of $93 \%$ and $88 \%$ when the DFT is increased to 256 points.

\section{CONCLUSION}

Simulation results for MMF links of $600 \mathrm{~m}, 1200 \mathrm{~m}$ and $2400 \mathrm{~m}$ have shown that the proposed combined receiver outperforms ZF receiver and can achieve a performance very close to the one given by V-BLAST with high savings in computational cost both in preprocessing as well as in detection stages. For example, when a 64-point DFT is used, the proposed receiver gives a complexity reduction of $86 \%$ in detection and $66 \%$ in preprocessing respect to the $\mathrm{ZF}$ receiver employed in [4].

The proposed combined receiver keeps the better performance of ZP-OFDM versus classic CP-OFDM in MMF links, but a reduced computational complexity is required when compared with other ZP-OFDM receivers.

\section{REFERENCES}

[1] Zizheng Cao, J. Yu, W. Wang, L. Chen, and Z. Dong, "Direct-Detection Optical OFDM Transmission System without Frequency Guard Band," IEEE Photon. Technol. Lett., vol. 22, no. 11, pp. 736-738, Jun. 1, 2010.

[2] R. P. Giddings, E. Hugues-Salas, B. Charbonnier, and J. M. Tang, "Experimental Demonstration of Real-Time Optical OFDM Transmission at $11.25 \mathrm{~Gb} / \mathrm{s}$ Over 500-m MMFs Employing Directly Modulated DFB
Lasers," IEEE Photon. Technol. Lett., vol. 23, no. 1, pp. 51-53, Jan. 1, 2011.

[3] X. Li, A. Alphones, W. Zhong, and C. Yu, "Investigation of PMD in direct-detection optical OFDM with zero padding," Optics Express, vol. 21, no 18, pp. 20851-20856, Sep. 2013.

[4] P. Medina, V. Almenar, and J. L. Corral, "Evaluation of optical ZP-OFDM transmission performance in multimode fiber links," Optics Express, vol. 22, no 1, pp. 1008-1017, Jan. 2014.

[5] C. Tepedelenlioglu, "Maximum Multipath Diversity with Linear Equalization in Precoded OFDM Systems," IEEE Trans. Inf. Theor., vol. 50, no 1, pp. 232-235, Jan. 2004.

[6] B. Muquet, Z. Wang, G. B. Giannakis, M. de Courville, and P. Duhamel, "Cyclic Prefixing or Zero Padding for Wireless Multicarrier Transmissions?," IEEE Trans. Commun., vol. 50, no 12, pp. 2136-2148, Dec. 2002.

[7] P. W. Wolniansky, G. J. Foschini, G. D. Golden, and R. A. Valenzuela, "V-BLAST: An Architecture for Realizing Very High Data Rates Over the Rich-Scattering Wireless Channel," in Proc. URSI Int. Symp. Signals, Systems, Electron., Pisa, Italy, Sep. 1998, pp 295-300.

[8] H. Zhu, W. Chen, B. Li, and F. Gao, "An Improved Square-Root Algorithm for V-BLAST Based on Efficient Inverse Cholesky Factorization," IEEE Trans. Wireless Commun., vol. 10, no 1, pp. 43-48, Jan. 2011.

[9] J. M. Tang, P. M. Lane, and K. A. Shore, "Transmission Performance of Adaptively Modulated Optical OFDM Signals in Multimode Fiber Links," IEEE Photon. Technol. Lett., vol. 18, no 1, pp. 205-207, Jan. 2006.

( a )

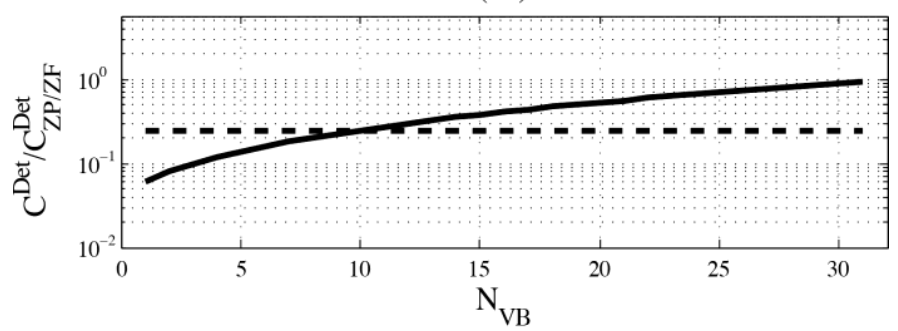

( b )

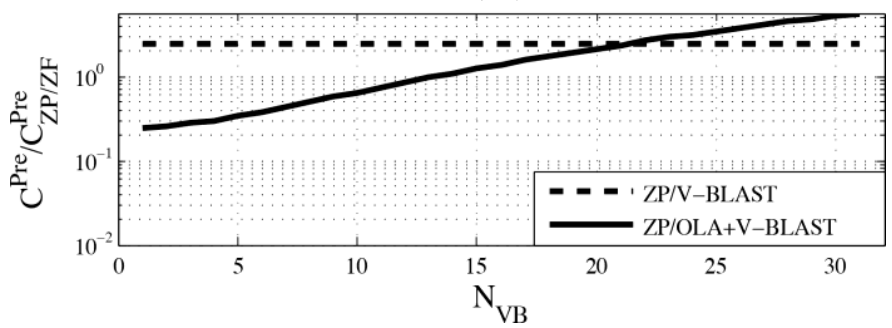

Fig. 3. Number of real multiplications required by detection stage (a) and preprocessing stage (b) for V-BLAST and OLA+V-BLAST ZP-OFDM receivers normalized with respect to $\mathrm{ZF}$, for a 64-points DFT.

TABLE I

COMPUTATIONAL COMPLEXITY COMPARISON

\begin{tabular}{||l|l|l|l|l|l|}
\hline$N_{F F T}$ & $N_{V B}$ & $\frac{C_{O L A+V B}^{D e t}}{C_{V B}^{D e t}}$ & $\frac{C_{O L A+V B}^{D e t}}{C_{Z F}^{D e t}}$ & $\frac{C_{O L A+V B}^{P r e}}{C_{V B}^{P r e}}$ & $\frac{C_{O L A+V B}^{P r e}}{C_{Z F}^{\text {Pre }}}$ \\
\hline \hline 64 & 5 & $56 \%$ & $14 \%$ & $14 \%$ & $34 \%$ \\
\hline 128 & 8 & $43 \%$ & $10 \%$ & $9 \%$ & $21 \%$ \\
\hline 256 & 12 & $30 \%$ & $7 \%$ & $6 \%$ & $12 \%$ \\
\hline
\end{tabular}

